\title{
Cerebrospinal Fluid Metals and the Association with Cerebral Small Vessel Disease
}

Mana Shams ${ }^{1} \mathrm{BS}$, Juha Martola ${ }^{1} \mathrm{MD}, \mathrm{PhD}$, Andreas Charidimou ${ }^{2} \mathrm{MD}, \mathrm{PhD}$, Tobias Granberg ${ }^{1}$, MD, PhD, Daniel Ferreira ${ }^{3} \mathrm{MD}, \mathrm{PhD}$, Eric Westman ${ }^{3} \mathrm{MD}, \mathrm{PhD}$, Maria Kristoffersen Wiberg ${ }^{1}$, MD, PhD, Niklas Forsgard ${ }^{4}$, PhD, Henrik Zetterberg ${ }^{5,6,7,8}, \mathrm{MD}, \mathrm{PhD}$, Lars-Olof Wahlund ${ }^{3} \mathrm{MD}$, PhD, Sara Shams ${ }^{1} \mathrm{MD}, \mathrm{PhD}$

${ }^{1}$ Department of Clinical Neuroscience, Karolinska Institutet, Stockholm, Sweden. Department of Radiology, Karolinska University Hospital, Stockholm, Sweden. (Address: same as corresponding author)

${ }^{2}$ Hemorrhagic Stroke Research Program, Department of Neurology, Massachusetts General Hospital Stroke Research Center, Harvard Medical School, Boston, MA, USA

${ }^{3}$ Department of Neurobiology, Care Sciences, and Society, Karolinska Institutet, Stockholm, Sweden. Division of Clinical Geriatrics, Karolinska University Hospital, Stockholm, Sweden. (Address: Karolinska Institutet, Department of Neurobiology, Care Sciences and Society, H1, Division of Clinical Geriatrics, Novum Floor 5, 141 86, Stockholm, Sweden)

${ }^{4}$ Clinical Chemistry Laboratory, Sahlgrenska University Hospital, Gothenburg, Swedem

${ }^{5}$ Clinical Neurochemistry Laboratory, Sahlgrenska University Hospital, Mölndal, Sweden

${ }^{6}$ Department of Psychiatry and Neurochemistry, Institute of Neuroscience and Physiology, the Sahlgrenska Academy at the University of Gothenburg, Mölndal, Sweden

${ }^{7}$ Department of Neurodgeneration, UCL Institute of Neurology, Queen Square, London, United Kingdsom

${ }^{8}$ UK Dementia Research Institute at UCL, London, United Kingdom

\section{Corresponding author:}

Sara Shams

Department of Radiology 
Karolinska University Hospital

SE-14186 Stockholm

E-mail: $\underline{\text { sara.shams@,ki.se }}$

Telephone number: +46735094299

Fax: +4687114840

\section{Running Title:}

Funding: Stockholm County Council, Karolinska Institutet, and the Swedish Dementia Association.

Disclosure: None for all authors. 


\begin{abstract}
Background:

Brain metal homeostasis is essential for brain health, and deregulation can result in oxidative stress on the brain parenchyma.
\end{abstract}

Objective:

Our objective in this study was to focus on two hemorrhagic MRI manifestations of small vessel disease [cerebral microbleeds (CMBs) and cortical superficial siderosis (cSS)] and associations with cerebrospinal fluid (CSF) iron levels. In addition, we aimed to analyze CSF biomarkers for dementia and associations with CSF metal levels.

Methods:

This is a cross-sectional study of 196 patients who underwent memory clinic investigation, including brain MRI. CSF was collected and analyzed for metals, amyloid- $\beta$ (A $\beta$ ) 42 , total tau (T-tau), and phosphorylated tau (P-tau), and CSF/serum albumin ratios. Statistical analyses were performed using generalized linear models.

Results:

No significant difference was found between CSF metal levels across diagnostic groups. Higher iron and copper levels were associated with higher CSF levels of A $\beta 42$, T-tau, P-tau, and $\mathrm{CSF} /$ serum albumin ratios $(\mathrm{p}<0.05)$. Zinc was associated with higher $\mathrm{CSF} / \mathrm{serum}$ albumin ratios. There was no significant association between CMBs or cSS and CSF iron levels. An increase in CSF iron with the number of CMBs was seen in APOE $\varepsilon 4$ carriers.

Conclusion: 
CSF iron levels are elevated with cerebral microbleeds in APOE $\varepsilon 4$ carriers, with no other association seen with hemorrhagic markers of small vessel disease. The association of elevated CSF iron and copper with tau could represent findings of increased neurodegeneration in these patients. 


\section{INTRODUCTION}

Iron has an essential role in the brain, being part of the synthesis of DNA, neurotransmitters and myelin, and serving as a cofactor in neuronal oxidative metabolism. ${ }^{1,2}$ With ageing the neuronal iron metabolism is thought to be increasingly deregulated, with iron depositing in the brain. ${ }^{1,2}$ Iron deposition in turn has long been suggested to be able to cause neurodegeneration, and be a causative factor for cognitive impairment and dementia. ${ }^{1-3}$ Iron deposition in the brain parenchyma is thought to promote neurodegeneration through inflammation and release of oxidative factors. ${ }^{1,2}$ Other metals such as zinc and copper have similarly been proposed to be involved in amyloid $\beta(A \beta) 42$ plaque formation, oxidative mechanisms and neurodegeneration.

Cerebral microbleeds (CMBs) and cortical superficial siderosis are markers of small vessel disease, common in cognitive impairment ${ }^{4,5}$. CMBs represent foci of hemosiderin deposits in the brain parenchyma. Cortical superficial siderosis in turn represent linear hemosiderin deposition in the cortex, the leptomeninges and within the subarachnoid space ${ }^{6}$. Cortical superficial siderosis has shown to be a sensitive marker for probable cerebral amyloid angiopathy, whereas CMBs in lobar locations are likely to be associated with cerebral amyloid angiopathy. Both these markers are readily seen on brain imaging with the use of susceptibility weighted imaging or T2* on MRI. Their presence has been suggested to lead to further neurodegenerative and cognitive decline.

In this study we hypothesized that iron deposition in the shape of cerebral microbleeds and cortical superficial siderosis would be reflected in CSF iron concentrations, with higher levels being associated with these markers. We also hypothesized that increased CSF metal concentrations would be associated with CSF biomarkers for plaque and tangle pathology (CSF amyloid $\beta 42$ [A $\beta 42]$ and phosphorylated tau [P-tau], respectively), as well as neurodegeneration (CSF total tau [T-tau]).

\section{MATERIAL AND METHODS}

\section{Patients}

This is a cross sectional study of a memory clinic population including 196 patients $(\mathrm{AD}=85$, mild cognitive impairment $=72$, subjective cognitive impairment $=32$, vascular dementia $=7$ ). 
All patients underwent memory clinic investigation, including detailed neuropsychological testing, blood tests, lumbar puncture with CSF analysis as well as an MRI brain scan. Patients were enrolled from January 2006 until January 2012, and were included on the basis of if they had CSF samples saved in a biobank for additional analysis. Exclusion criterion was insufficient quality of the MRI scan, with no exclusions in our cohort.

\section{MRI Protocol and Image Analysis}

All patients had a full MRI protocol including hemosiderin sensitive MRI sequences, the T2* and/or the susceptibility weighted imaging sequence. Image analysis was done according to the STRIVE criteria ${ }^{7}$, by a neuroradiologist and an experienced $\mathrm{MD}, \mathrm{PhD}$ as prior described ${ }^{5}$.

\section{CSF Analysis and Apolipoprotein E}

Initial CSF analysis was done as previously described (REF) and included routine CSF biomarker analysis: amyloid $\beta$ (A $\beta 42)$, total tau (T-tau), phosphorylated tau (P-tau) and CSF serum albumin ratio. Additional analyses were done on CSF samples saved in a biobank. In this study we focused on iron-related markers and iron concentration in the CSF was specifically measured. Apolipoprotein E ( $A P O E)$ genotyping was done on a sub-cohort $(\mathrm{n}=102)$.

\section{Statistical analysis}

SPSS 24.0 was used for statistical analysis. CSF metal concentrations as well as CSF biomarkers were log transformed, when appropriate to obtain normality. ANOVAs were used to assess differences in CSF metal concentrations amongst groups. Linear regression models were built to assess associations between CSF metals and CSF biomarkers with results presented as the standardized regression coefficient $\beta$. Clinical associations were tested in negative binomial regression models (results presented with the unstandardized regression coefficient B) as well as binary logistic regression models. Negative binomial regression models were built, adding an interaction between $A P O E \& 4$ and iron concentrations. The threshold for statistical significance was set as $\mathrm{P}<0.05$, and was after accounting for multiple testing according to the Benjamini-Hochberg procedure set to $\mathrm{P}<0.02^{8}$.

\section{RESULTS}

A total of 196 patients were recruited for our study, with demographics presented as in Table

1. CSF concentrations of iron, manganese, copper, cobolt and zinc did not differ between 
diagnostic groups. Chromium was higher in $\mathrm{AD}$ than $\mathrm{SCI}(\mathrm{P}=0.003)$. MCI had higher chromium levels than SCI $(\mathrm{P}<0.001)$ and vascular dementia $(\mathrm{P}=0.012)$. Nickel was higher in MCI compared to $\mathrm{AD}, \mathrm{VaD}$ and $\mathrm{SCI}(\mathrm{P}=0.001)$ and higher in $\mathrm{AD}$ compared to $\mathrm{VaD}$ and $\mathrm{SCI}$ $(\mathrm{P}<0.001)$.

Exploring the relationships of CSF metals with CSF biomarkers for AD pathology showed an association between A 442 and higher iron and copper levels in the CSF (Table 2). Higher levels of chromium, manganese, iron, and copper were associated with higher levels of T-tau. Higher levels of iron and copper were associated with higher levels of P-tau. A disrupted blood-brain barrier, reflected by the $\mathrm{CSF} /$ serum albumin ratio, was associated with higher levels of iron, copper and zinc deposition in the brain. No associations were seen between CSF metals and hypertension, MMSE score or age. CSF AD biomarkers and iron, copper and zinc were further analyzed in detail in the diagnostic groups (Table 3). Increased A $\beta 42$ levels were associated with increased level of iron and copper in AD and MCI, and only with zinc in AD. T-tau increased with increasing iron and copper concentrations in MCI and SCI. Similarly, P-tau increased with increasing iron concentrations in MCI, and showed a tendency to do so in SCI and AD. P-tau also increased with increasing copper concentrations in AD and MCI. Increased $\mathrm{CSF} /$ serum albumin ratio was associated with increased CSF iron and copper concentrations, as well as zinc in AD and MCI.

CMBs did not show any association to iron levels. When looking at interactions with APOE $\varepsilon 4$, carriers had a higher iron level concentration associated with increasing number of CMBs (Table 4). Cortical superficial siderosis showed no association with iron levels in the whole cohort and in the diagnostic groups even when adding interactions with ApoE $\varepsilon 4$. There was no difference in iron concentrations between patients with and without probable CAA, classified according to CMB location.

\section{DISCUSSION}

Metals are higher in the CSF with neurodegeneration, and are associated with a disrupted blood brain barrier. Metals were associated with lesser amyloid deposition in the brain, reflected by 
higher $A \beta 42$ levels. This suggests the impact, and possible role of metals in neurodegenerative disorders. Increased iron levels were associated with increased CMBs in $A P O E$ \&4 carriers.

Metals hold an important role in several important biological processes and a regulated metal homeostasis is of the essence. In the brain iron contributes to cell growth, enzymatic activities, immune responses and oxygenation ${ }^{9}$, and other metals have equally essential roles.

Metal dyshomeostasis has been hypothesized to cause damage, amongst others, through oxidative stress, interaction with DNA, proteins and other macromolecules ${ }^{10}$. Oxidative stress has been proposed to be one of the earliest mechanisms on the path to MCI and $\mathrm{AD}^{11,12}$. The creation of reactive oxygen species may lead to interaction with cell signaling, and DNA disruption as well as cell apoptosis, and neuronal damage ${ }^{10}$. Iron and copper are also highly involved in the $\mathrm{A} \beta 42$ aggregation in $\mathrm{AD}$ and may further thus contribute to the disease process ${ }^{13}$. Copper has equally shown to cause reactive oxygen species, and is thought to interact with amyloid precursor protein promoting the production of $\mathrm{A} \beta 42$ plaques and neurofibrillary tangles ${ }^{11}$.

Prior studies on CSF metals and neurodegeneration are scarce, and especially that of the interrelation with small vessel disease were none exist to date. Small vessel disease has been hypothesized to be able to lead to dementia, and the fragile leaking microvasculature leading to CMBs representing an additional source of hemosiderin in the brain parenchyma. CMBs have shown to be associated with amyloid brain deposition, ${ }^{14}$ supporting the intriguing theory that the $\mathrm{CMB}$ in itself may trigger $\mathrm{A} \beta 42$ plaque formation.

Iron has been widely studied in neurodegeneration. In a case report, superficial CNS siderosis with iron toxicity was associated with increased CSF T-tau and P-tau concentrations, which 
may reflect Alzheimer-like neurodegeneration, whilst CSF A $\beta 42$ was normal (i.e., high), suggesting no cerebral $\beta$-amyloidosis (PMID: PMID: 18617192). MCI and AD have been shown to have a higher load of iron in the brain parenchyma on histopathology ${ }^{11}$. Hippocampal atrophy rate has shown to be higher in patients with higher CSF ferritin levels ${ }^{15}$. CSF ferritin levels have also shown to be associated with cognition, as well as prediction of conversion from $\mathrm{MCI}$ to $\mathrm{AD}^{15} . A P O E \varepsilon 4$ has shown to be associated with higher CSF ferritin levels, indicating a possible pathway of pathology of the allele ${ }^{15}$. In our study, high iron levels were associated with increased T-tau and P-tau, as well as a disrupted blood-brain barrier, reflected by the $\mathrm{CSF} / \mathrm{serum}$ albumin ratio. The association between iron and high $\mathrm{A} \beta 42$ is not entirely unexpected, considering what is known about superficial CNS siderosis, and may indicate that oxidation is not associated with $\mathrm{A} \beta 42$ aggregation in the brain, but mainly with neurodegeneration and neurofibrillary tangles. Iron levels increased with number of CMBs in $A P O E \varepsilon 4$ carriers.

Free copper has also shown to be higher in patients with $\mathrm{AD}$, and to be able to predict conversion from MCI to $\mathrm{AD}^{16}$. CSF copper levels have also shown to be higher in patients with $\mathrm{AD}$, and especially with late onset $\mathrm{AD}$, when compared with controls ${ }^{17}$. The importance of copper is supported in our study with the associations to biomarkers for neurofibrillary tangles, neurodegeneration and blood brain barrier disruption. However, similar to iron, copper was associated with higher $A \beta 42$ levels in the CSF.

Zinc has shown to be enriched in $A \beta 42$ plaques $^{11,18}$. The $A \beta 42$ forming process is thought to deplete neurons from the intracellular zinc storage, leading to microtubule disarray as well as formation of neurofibrillary tangles ${ }^{11,18}$. CSF zinc has shown to be higher with late onset AD, albeit in a cohort with only 14 participants ${ }^{17}$. A higher level of zinc was associated with blood- 
brain barrier disruption as well as a higher $\mathrm{A} \beta 42$ in $\mathrm{AD}$ in our study. The association with $\mathrm{A} \beta$ 42 may once again indicate that pathomechanistic effects of zinc take place in an early stage, and are not specific to AD.

Other metals including chromium, manganese, cobalt and nickel are scarcely studied in neurodegeneration. In a similar study to ours on CSF in $\mathrm{AD}$, cobalt showed to be the only metal that was higher in $\mathrm{AD}$ than in healthy controls, and this held true when only looking at a sub cohort with severe $\mathrm{AD}^{19}$. No significant associations between these metals and biomarkers of dementia were seen in our study.

Limitations of our study include the fact that CSF metal levels may not accurately depict that of the brain parenchyma ${ }^{20}$ - however our analysis may reflect the condition of the brain parenchyma, and postmortem analysis may not always reflect that of the living brain. Quantitative susceptibility mapping provides an additional way of in vivo iron quantification, and will be used in our further studies. Benefits include a wide array of metal analysis in a large cohort, with imaging analysis.

Iron, copper and zinc appear to be of importance in neurodegeneration, but are not associated with higher $\mathrm{A} \beta 42$ levels in the brain. Iron levels increase with $\mathrm{CMBs}$ in $A P O E \varepsilon 4$ carriers only.

\section{REFERENCES}

1. Acosta-Cabronero, J., Betts, M. J., Cardenas-Blanco, A., Yang, S. \& Nestor, P. J. In Vivo MRI Mapping of Brain Iron Deposition across the Adult Lifespan. J. Neurosci. 36, 364-374 (2016).

2 .

Daugherty, A. M., Haacke, E. M. \& Raz, N. Striatal Iron Content Predicts Its Shrinkage and Changes in Verbal Working Memory after Two Years in Healthy Adults. J. Neurosci. 35, 6731 (2015).

3. Penke, L. et al. Brain iron deposits are associated with general cognitive ability and cognitive aging. Neurobiol. Aging 33, 510-517.e2 (2012).

4. Shams, S. et al. Cerebral microbleeds: different prevalence, topography, and risk factors 
depending on dementia diagnosis - the Karolinska Imaging Dementia Study. AJNR Am. J. Neuroradiol. 36, 661666 (2015).

5. Shams, S. et al. Cortical superficial siderosis: Prevalence and biomarker profile in a memory clinic population. Neurology 87, 1110-1117 (2016).

6. Charidimou, A. et al. Cortical superficial siderosis: detection and clinical significance in cerebral amyloid angiopathy and related conditions. Brain J. Neurol. 138, 2126-2139 (2015).

7. Wardlaw, J. M. et al. Neuroimaging standards for research into small vessel disease and its contribution to ageing and neurodegeneration. Lancet Neurol. 12, 822-838 (2013).

8. Benjamini, Y. \& Hochberg, Y. Controlling the false discovery rate: A practical and powerful approach to multiple testing. Journal of the Royal Statistical Society, Series B: Methodological. 1995, 289-300

9. Jomova, K. \& Valko, M. Advances in metal-induced oxidative stress and human disease. Toxicology 283, 65-87 (2011).

10. Cicero, C. E. et al. Metals and neurodegenerative diseases. A systematic review. Environ. Res. 159, 82-94 (2017).

11. Smith, M. A. et al. Increased Iron and Free Radical Generation in Preclinical Alzheimer Disease and Mild Cognitive Impairment. J. Alzheimers Dis. 19, 363-372 (2010).

12. Smith, M. A. Oxidative stress and iron imbalance in Alzheimer disease: how rust became the fuss! J. Alzheimers Dis. JAD 9, 305-308 (2006).

13. Mattson, M. P. Pathways towards and away from Alzheimer's disease. Nature 430, 631-639 (2004).

14.

Park, J.-H. et al. Pathogenesis of cerebral microbleeds: In vivo imaging of amyloid and subcortical ischemic small vessel disease in 226 individuals with cognitive impairment. Ann. Neurol. 73, 584-593 (2013).

15. Ayton, S., Faux, N. G. \& Bush, A. I. Ferritin levels in the cerebrospinal fluid predict Alzheimer's disease outcomes and are regulated by APOE. Nat. Commun. 6, (2015).

16. Squitti, R. et al. Value of serum nonceruloplasmin copper for prediction of mild cognitive impairment conversion to Alzheimer disease. Ann. Neurol. 75, 574-580 (2014).

17. Hozumi, I. et al. Patterns of levels of biological metals in CSF differ among neurodegenerative diseases. J. Neurol. Sci. 303, 95-99 (2011).

18. Craddock, T. J. A. et al. The Zinc Dyshomeostasis Hypothesis of Alzheimer's Disease. PLOS ONE 7, e33552 (2012).

19. Gerhardsson, L., Lundh, T., Londos, E. \& Minthon, L. Cerebrospinal fluid/plasma quotients of essential and non-essential metals in patients with Alzheimer's disease. J. Neural Transm. Vienna Austria 1996 118, 957-962 (2011).

20. Szabo, S. T., Harry, G. J., Hayden, K. M., Szabo, D. T. \& Birnbaum, L. Comparison of Metal Levels between Postmortem Brain and Ventricular Fluid in Alzheimer's Disease and Nondemented Elderly Controls. Toxicol. Sci. 150, 292-300 (2016). 
Table 1. Patient demographics.

\begin{tabular}{|c|c|c|c|c|}
\hline & AD $(n=85)$ & $\operatorname{MCI}(n=72)$ & $\operatorname{VaD}(n=7)$ & $\operatorname{SCI}(n=32)$ \\
\hline Age, $\operatorname{mean} \pm$ SD & $66 \pm 8$ & $63 \pm 8$ & $65 \pm 11$ & $57 \pm 8$ \\
\hline Gender, F/M \% & $55 / 45$ & $33 / 67$ & $57 / 43$ & $53 / 47$ \\
\hline MMSE, mean \pm SD & $23 \pm 5$ & $26 \pm 4$ & $24 \pm 4$ & $28 \pm 2$ \\
\hline CMB, n \% & $28(33)$ & $27(38)$ & $4(57)$ & $14(44)$ \\
\hline cSS, n \% & $8(9)$ & $3(4)$ & 0 & 0 \\
\hline CSF iron, median IQR & $16(13-19)$ & $16(13-20)$ & $16(13-17)$ & $16(14-20)$ \\
\hline $\begin{array}{l}\text { CSF chromium, } \\
\text { median IQR }\end{array}$ & $0.87(0.74-0.11)^{\mathrm{a}}$ & $0.89(0.74-0.11)^{b}$ & $0.54(0.54-0.54)^{b}$ & $\begin{array}{l}0.58 \\
0.70)^{\mathrm{a}, \mathrm{b}}\end{array}$ \\
\hline $\begin{array}{l}\text { CSF manganese, } \\
\text { median IQR }\end{array}$ & $0.41(0.34-0.50)$ & $0.42(0.34-0.51)$ & $0.30(0.29-0.39)$ & $0.42(0.36-0.49)$ \\
\hline $\begin{array}{ll}\text { CSF cobolt, median } \\
\text { IQR }\end{array}$ & $0.08(0.08-0.08)$ & $0.08(0.08-0.08)$ & $0.08(0.08-0.08)$ & $0.08(0.08-0.08)$ \\
\hline $\begin{array}{ll}\text { CSF nickel, median } \\
\text { IQR }\end{array}$ & $\begin{array}{l}0.11 \\
0.11)^{\mathrm{c}, \mathrm{d}}\end{array}$ & $\begin{array}{l}0.11(0.11- \\
0.16)^{\mathrm{c}}\end{array}$ & $\begin{array}{l}0.11 \\
0.11)^{\mathrm{c}, \mathrm{d}}\end{array}$ & $\begin{array}{l}0.11 \\
0.11)^{\mathrm{c}, \mathrm{d}}\end{array}$ \\
\hline $\begin{array}{l}\text { CSF cupper, median } \\
\text { IQR }\end{array}$ & $10.7(9.2-12.4)$ & $10.8(9.6-12.4)$ & $10.59(8.4-15.3)$ & $12(10.9-12.6)$ \\
\hline CSF zinc, median IQR & $12.6(11-16)$ & $13.5(11-17)$ & $12(9-14)$ & $13(12-15)$ \\
\hline A $\beta$ 42, median IQR & $482(414-586)$ & $732(500-975)$ & $994(618-1170)$ & $1115(974-1288)$ \\
\hline
\end{tabular}

${ }^{\mathrm{a}} \mathrm{P}<0.05$ between $\mathrm{AD}$ and $\mathrm{SCI},{ }^{b} \mathrm{P}<0.05$ between $\mathrm{MCI}$ and $\mathrm{SCI}$, and $\mathrm{MCI}$ and $\mathrm{VaD} .{ }^{\mathrm{c}} \mathrm{P}<0.05$, between $\mathrm{MCI}$ and $\mathrm{AD}, \mathrm{MCI}$ and SCI, MCI and $\mathrm{VaD} .{ }^{\mathrm{d}} \mathrm{P}<0.05$ between $\mathrm{AD}$ and $\mathrm{Vad}$ and $\mathrm{AD}$ and $\mathrm{SCI}$. AD = Alzheimer's disease, $\mathrm{IQR}=$ Interquartile range, $\mathrm{MCI}=$ Mild cognitive impairment, $\mathrm{SCI}=$ Subjective cognitive impairment, $\mathrm{VaD}=$ Vascular dementia. 
Table 2. Clinical associations with CSF metals.

\begin{tabular}{|c|c|c|c|c|c|c|c|}
\hline & $\begin{array}{l}\text { CHROMIU } \\
\text { M }\end{array}$ & $\begin{array}{l}\text { MANGANES } \\
\text { E }\end{array}$ & IRON & $\begin{array}{l}\text { COBAL } \\
T\end{array}$ & $\begin{array}{l}\text { NICKE } \\
\text { L }\end{array}$ & $\begin{array}{l}\text { COPPE } \\
\text { R }\end{array}$ & ZINC \\
\hline $\begin{array}{l}\text { AMYLOID } \\
\text { BETA } 42\end{array}$ & $-0.055, \mathrm{P}=0.4$ & $-0.09, \mathrm{P}=0.23$ & $\begin{array}{l}\mathbf{0 . 2 1}, \\
P=0.00 \\
4\end{array}$ & $\begin{array}{l}-0.085, \\
\mathrm{P}=0.24\end{array}$ & $\begin{array}{l}0.002, \\
\mathrm{P}=0.97\end{array}$ & $\begin{array}{l}\text { 0.23, } \\
P=0.001\end{array}$ & $\begin{array}{l}0.104, \\
\mathrm{P}=0.14 \\
8\end{array}$ \\
\hline T-TAU & $0.133, P=0.06$ & $0.13, P=0.08$ & $\begin{array}{l}0.27 \\
P<0.00 \\
1\end{array}$ & $\begin{array}{l}0.1, \\
\mathrm{P}=0.16\end{array}$ & $\begin{array}{l}0.04, \\
\mathrm{P}=0.63\end{array}$ & $\begin{array}{l}\text { 0.23, } \\
P=0.001\end{array}$ & $\begin{array}{l}0.08, \\
\mathrm{P}=0.3\end{array}$ \\
\hline P-TAU & $0.10, \mathrm{P}=0.16$ & $0.13, \mathrm{P}=0.07$ & $\begin{array}{l}\mathbf{0 . 3 0}, \\
\mathbf{P}<\mathbf{0 . 0 0} \\
1\end{array}$ & $\begin{array}{l}0.11 \\
\mathrm{P}=0.14\end{array}$ & $\begin{array}{l}0.19 \\
\mathrm{P}=0.79\end{array}$ & $\begin{array}{l}\text { 0.26, } \\
P<0.001\end{array}$ & $\begin{array}{l}0.08, \\
\mathrm{P}=0.28 \\
0\end{array}$ \\
\hline $\begin{array}{l}\text { CSF/serum } \\
\text { albumin } \\
\text { ratio }\end{array}$ & $0.02, \mathrm{P}=0.80$ & $-0.12, \mathrm{P}=0.09$ & $\begin{array}{l}0.52, \\
P<0.00 \\
1\end{array}$ & $\begin{array}{l}0.06, \\
\mathrm{P}=0.40\end{array}$ & $\begin{array}{l}0.03, \\
\mathrm{P}=0.68\end{array}$ & $\begin{array}{l}\text { 0.65, } \\
P<0.001\end{array}$ & $\begin{array}{l}0.17, \\
P=0.02\end{array}$ \\
\hline MMSE & $\begin{array}{l}-0.001 \\
\mathrm{P}=0.603\end{array}$ & $\begin{array}{l}-0.001, \\
\mathrm{P}=0.799\end{array}$ & $\begin{array}{l}0.002, \\
\mathrm{P}=0.85\end{array}$ & $\begin{array}{l}-0.03 \\
\mathrm{P}=0.86\end{array}$ & $\begin{array}{l}-0.001, \\
\mathrm{P}=0.803\end{array}$ & $\begin{array}{l}0.002, \\
\mathrm{P}=0.936\end{array}$ & $\begin{array}{l}0.001, \\
\mathrm{P}=0.93\end{array}$ \\
\hline Age & $0.0, \mathrm{P}=0.742$ & $0.0, \mathrm{P}=0.985$ & $\begin{array}{l}0.006, \\
P=0.67 \\
3\end{array}$ & $\begin{array}{l}0.02, \\
\mathrm{P}=0.903\end{array}$ & $\begin{array}{l}0.0 \\
\mathrm{P}=0.87\end{array}$ & $\begin{array}{l}0.07 \\
\mathrm{P}=0.761\end{array}$ & $\begin{array}{l}0.002, \\
\mathrm{P}=0.88 \\
7\end{array}$ \\
\hline $\begin{array}{l}\text { Hypertensio } \\
\text { n }\end{array}$ & $\begin{array}{l}-0.01 \\
P=0.037\end{array}$ & $0.007, \mathrm{P}=0.554$ & $\begin{array}{l}0.009, \\
\mathrm{P}=0.74 \\
5\end{array}$ & $3.2, \mathrm{P}=1.0$ & $\begin{array}{l}-0.01, \\
P=0.18\end{array}$ & $\begin{array}{l}0.0 \\
\mathrm{P}=0.697\end{array}$ & $\begin{array}{l}0.005, \\
\mathrm{P}=0.85\end{array}$ \\
\hline
\end{tabular}

Values are given as the standardized regression coefficient beta and the nonstandardized regression coefficient for MMSE, Age and Hypertension. 
Table 3. CSF iron, copper and zinc levels within the separate diagnostic groups.

\begin{tabular}{|l|l|l|l|}
\hline$\underline{\text { Iron }}$ & $\mathbf{A D}$ & $\mathbf{M C I}$ & $\mathbf{S C I}$ \\
\hline $\mathbf{A} \boldsymbol{\beta} 42$ & $\mathbf{0 . 3 1}, \mathbf{P}=\mathbf{0 . 0 0 5}$ & $\mathbf{0 . 2 7}, \mathbf{P}=\mathbf{0 . 0 2}$ & $0.16, \mathrm{P}=0.391$ \\
\hline T-tau & $0.15, \mathrm{P}=0.17$ & $\mathbf{0 . 4 6}, \mathbf{P}<\mathbf{0 . 0 0 1}$ & $\mathbf{0 . 5 7}, \mathbf{P}=\mathbf{0 . 0 0 1}$ \\
\hline P-tau & $0.21, \mathrm{P}=0.06$ & $\mathbf{0 . 4 5}, \mathbf{p}<\mathbf{0 . 0 0 1}$ & $\mathbf{0 . 3 6}, \mathbf{p}=\mathbf{0 . 0 4 5}$ \\
\hline CSF/serum alb & $\mathbf{0 . 5 8}, \mathbf{p}<\mathbf{0 . 0 0 1}$ & $\mathbf{0 . 4 6}, \mathbf{p}<\mathbf{0 . 0 0 1}$ & $\mathbf{0 . 3 8}, \mathbf{p}=\mathbf{0 . 0 3}$ \\
\hline Copper & & & \\
\hline A $\boldsymbol{\beta} 42$ & $\mathbf{0 . 2 6}, \mathbf{P}=\mathbf{0 . 0 1}$ & $\mathbf{0 . 2 9}, \mathbf{P}=\mathbf{0 . 0 1}$ & $0.14, \mathrm{P}=0.45$ \\
\hline T-tau & $0.18, \mathrm{P}=0.09$ & $\mathbf{0 . 4 2}, \mathbf{P}<\mathbf{0 . 0 0 1}$ & $\mathbf{0 . 4 4}, \mathbf{P}=\mathbf{0 . 0 1}$ \\
\hline $\mathbf{P}$-tau & $\mathbf{0 . 2 7}, \mathbf{P}=\mathbf{0 . 0 1}$ & $\mathbf{0 . 3 7}, \mathbf{P}=\mathbf{0 . 0 0 1}$ & $0.17, \mathrm{P}=0.36$ \\
\hline CSF/serum alb & $\mathbf{0 . 6 3}, \mathbf{P}<\mathbf{0 . 0 0 1}$ & $\mathbf{0 . 6 4}, \mathbf{P}<\mathbf{0 . 0 0 1}$ & $\mathbf{0 . 6 5}, \mathbf{P}<\mathbf{0 . 0 0 1}$ \\
\hline Zinc & & & \\
\hline A $\boldsymbol{\beta} 42$ & $\mathbf{0 . 4 0 ,} \mathbf{P}<\mathbf{0 . 0 0 1}$ & $-0.0, \mathrm{P}=0.99$ & $0.19, \mathrm{P}=0.29$ \\
\hline T-tau & $-0.02, \mathrm{P}=0.83$ & $0.19, \mathrm{P}=0.11$ & $0.37, \mathrm{P}=0.03$ \\
\hline P-tau & $0.03, \mathrm{P}=0.74$ & $0.15, \mathrm{P}=0.21$ & $-0.00, \mathrm{P}=0.99$ \\
\hline CSF/serum alb & $\mathbf{0 . 3 1}, \mathbf{P}=\mathbf{0 . 0 0 5}$ & $\mathbf{0 . 0 0 9}, \mathbf{P}<\mathbf{0 . 0 0 1}$ & $0.20, \mathrm{P}=0.09$ \\
\hline
\end{tabular}

Values are given as the standardized regression coefficient beta. 
Table 4. Cerebral microbleeds and associations with iron concentration.

\begin{tabular}{|l|l|l|l|l|}
\hline & $\begin{array}{l}\text { Whole } \\
\text { cohort }\end{array}$ & AD & MCI & SCI \\
\hline Presence of CMBs & $0.14 \mathrm{P}=0.09$ & $0.14, \mathrm{p}=0.21$ & $0.20, \mathrm{p}=0.09$ & $-0.06, \mathrm{p}=0.76$ \\
\hline Number of CMBs & $0.12 \mathrm{P}=0.09$ & $0.15, \mathrm{p}=0.16$ & $0.12, \mathrm{p}=0.30$ & $-0.04, \mathrm{p}=0.82$ \\
\hline $\begin{array}{l}\text { Number of CMBs if } \\
\text { ApoE } \varepsilon 4 \text { carrier }\end{array}$ & $\mathbf{0 . 1 2}, \mathbf{P}=\mathbf{0 . 0 1}$ & $0.06, \mathrm{P}=0.14$ & $\mathbf{0 . 3 2}, \mathbf{P}<\mathbf{0 . 0 0 1}$ & $-0.09, \mathrm{P}=0.54$ \\
\hline
\end{tabular}

Values are given as the regression coefficient B. 